\title{
Exploring coherent transport through $\pi-$ stacked systems for molecular electronic devices
}

\author{
Qian Li and Gemma C. Solomon* \\ Received 28th April 2014, Accepted 2nd June 2014 \\ DOI: $10.1039 / \mathrm{c} 4 \mathrm{fd} 00083 \mathrm{~h}$
}

\begin{abstract}
Understanding electron transport across $\pi$-stacked systems can help to elucidate the role of intermolecular tunneling in molecular junctions and potentially with the design of highefficiency molecular devices. Here we show how conjugation length and substituent groups influence the electron transport and thermoelectric response in the $\pi$-stacked structures by investigating five representative stacked molecular junctions. We found that a $\pi$-stacked system of two substituted anthracenes exhibits good thermopower and a high power factor, suggesting that increased conjugation can enhance the thermoelectric response. The fully eclipsed structure of quinhydrone exhibits a high power factor at the minimum energy structure and could thus be a better candidate in a thermoelectric device compared with the other $\pi$-stacked systems considered.
\end{abstract}

\section{Introduction}

The starting point for the chemists' vision of molecular electronics is a single molecule bound between electrodes, where chemical functionality within the molecule has been carefully designed to yield a particular electrical function. While such a design sounds simple, achieving this goal has been anything but simple. Leaving aside for a moment the challenge of developing a particular electrical function from chemical functionality, it is a nontrivial task to bind a single molecule between electrodes. Most of the molecules targeted have been large conjugated molecules and consequently aggregation is always a possibility. Within the junction, $\pi$-stacked structures can form by accident or by design. Within such structures, there is always the possibility of intermolecular tunnelling, introducing a degree of ambiguity in the interpretation of the results. The role of intermolecular $\pi-\pi$ interactions in mediating transport has not been extensively explored and warrants further investigation.

$\pi-\pi$ stacking is efficiently used by nature to achieve directed long-range electron transport, such as DNA base pairs ${ }^{\mathbf{1 , 2}}$ and amino acid residues. Careful 
experiments have found that the intermolecular $\pi-\pi$ stacking interaction can form an electrical junction and lead to molecular junctions with significant conductance. ${ }^{3,4}$ An STM break-junction experiment also measured single-molecule conductance through $\pi-\pi$ stacked benzene rings and found hydrocarbons form direct electrical contacts with gold electrodes. ${ }^{5}$ Understanding electron transport across $\pi$-stacked structures will help to elucidate the role of intermolecular tunneling in the molecular junction and allow us to use these interactions to design better molecular devices.

Returning to the other challenge in this problem, achieving particular electrical functionality, there has been considerable interest in recent years in quantum interference as a means to make conjugated molecules more than just wires. Destructive quantum interference has been shown to occur in $\pi$ stacked systems and can be modulated with changing spatial dislocation between the stacked elements. ${ }^{6,7}$ Beyond traditional electrical components, there has also been an interest in molecular thermoelectrics. Thermoelectric effects may be dramatically enhanced near the transmission node that arises as a result of this destructive interference. ${ }^{8}$ Several groups have reported the measurement of thermopower, $S$, of molecular junctions. ${ }^{9-13}$ The thermopower enters the figure of merit, $Z T=S^{2} G T / \kappa$, which is used to describe the efficiency of a thermoelectric device. Here $S$ is the thermopower, $G$ is the electronic conductance, $T$ is temperature and $\kappa$ is the thermal conductance with contribution from electrons and phonons. As $\pi$-stacked systems are electronically coupled through-space, we anticipate that the thermal conductance will be low. Consequently, in this situation the magnitude of the figure of merit is mainly determined by the power factor $S^{2} G$. Although the conductance is low at energies where destructive interference dominates, the power factor $\left(S^{2} G\right)$ can have a high value in the case where $S^{2}$ is much larger than $G$. In the case where an interference feature is in close proximity to a resonant peak (for example a fano-resonance), however, it is possible to obtain both high thermopower and relatively high conductance.

In this paper, we investigate electron transport and the thermoelectric response through five representative $\pi$-stacked systems (three systems with differing conjugation length and two systems with different substituents). For each system, the total transmission at the selected geometries is calculated to illustrate the change in transmission with the spatial dislocation. We examine how conjugation length and substituents influence the electronic transmission, thermopower and power factor in these $\pi$-stacked systems. Finally, the binding energy is calculated to show the differing stability of the structures.

The five $\pi$-stacked systems considered in this paper are shown in Fig. 1 . As we expect an edge to face structure to have weak electronic coupling, resulting in low conductance, we only consider the face to face structure for $\pi$-stacked dimers in our study. The three systems A, B and C are $\pi$-stacked systems with different conjugation lengths. System D has a substituted cyano group and system E comprises a quinone and a hydroquinone. All of the junctions are formed by one molecule chemisorbed on the left electrode, and another one chemisorbed on the right electrode, as shown at the bottom of Fig. 1. 


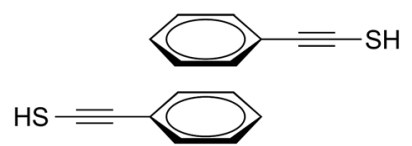

A

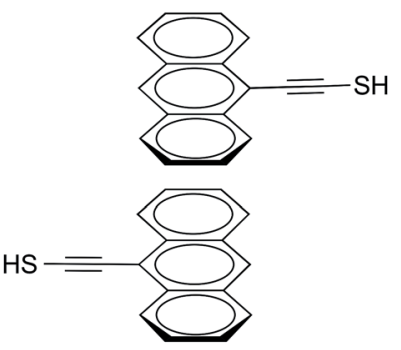

B

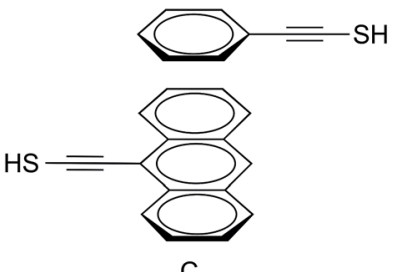

Systems with substituents

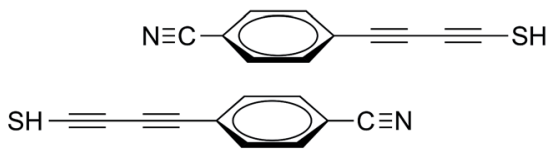

D
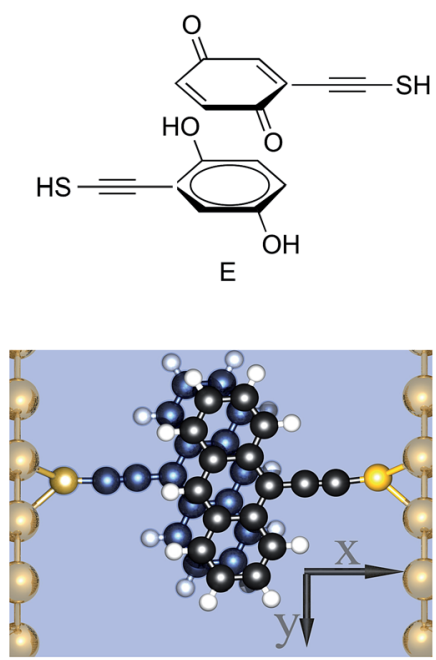

Fig. 1 Five different $\pi$-stacked molecules we consider in this work. A, B and C are $\pi$ stacked systems without substituents while system $D$ is substituted with cyano groups and system $E$ is the combination of a quinone and a hydroquinone. Bottom (right): an illustration of $B$ when chemisorbed in between gold electrodes as used in our transport calculations.

\section{Background}

As inelastic transport is usually strongly suppressed at room temperature in single molecule junctions, we only consider the elastic transport. The current through a molecule is calculated with a Green's function implementation of the Landauer approach: ${ }^{14}$

$$
I(V)=\frac{2 e}{h} \int_{-\infty}^{\infty} \mathrm{d} E\left[f_{\mathrm{L}}(E, V)-f_{\mathrm{R}}(E, V)\right] T(E, V)
$$

where $f_{\mathrm{L}}(E, V)$ and $f_{\mathrm{R}}(E, V)$ are the Fermi functions for the left and right electrodes, $T(E, V)$ is the energy and bias dependent transmission. Here, we report the transmission that underlies the zero-bias conductance. The Fermi energy is set to $-5 \mathrm{eV}$. The thermopower is calculated by:

$$
S=-\left.\frac{\pi^{2} k_{\mathrm{B}}^{2} T}{3 e T(E)} \frac{\partial T(E)}{\partial E}\right|_{E=E_{\mathrm{F}}}
$$


This equation indicates that a high thermopower is achieved when the slope of the transmission is steep.

\section{Computational methods}

The geometries of the isolated molecules were optimized using GPAW ${ }^{15}$ (a gridbased real-space DFT code) using PBE exchange functional with van der Waals correction (TS09) ${ }^{16}$ and the stacked structures were formed by combining two of these structures to calculate the transport and binding energy. The moleculemolecule separation in the $z$-direction (perpendicular to the molecular plane) was set to the value that gave the minimum energy structure. The molecules were chemisorbed on an FCC hollow site of the Au electrodes with terminal hydrogen atoms removed. All the transport calculations were performed using gDFTB $^{17,18}$ with the geometries obtained from GPAW.

\section{Results and discussion}

\subsection{System A}

We start by considering a basic system to illustrate the basic properties of the transmission and thermoelectric response: stacked benzene rings with thiol substituents for binding to gold electrodes. The transmission features can be understood by considering the system as an even alternate hydrocarbon. ${ }^{19}$ Fig. 2 (top) illustrates how the starring assignment works in the $\pi$-stacked systems: mark every second atom with a star and continue across the nearest-neighbour interactions in the stack. When the two sulphur atoms are both marked with
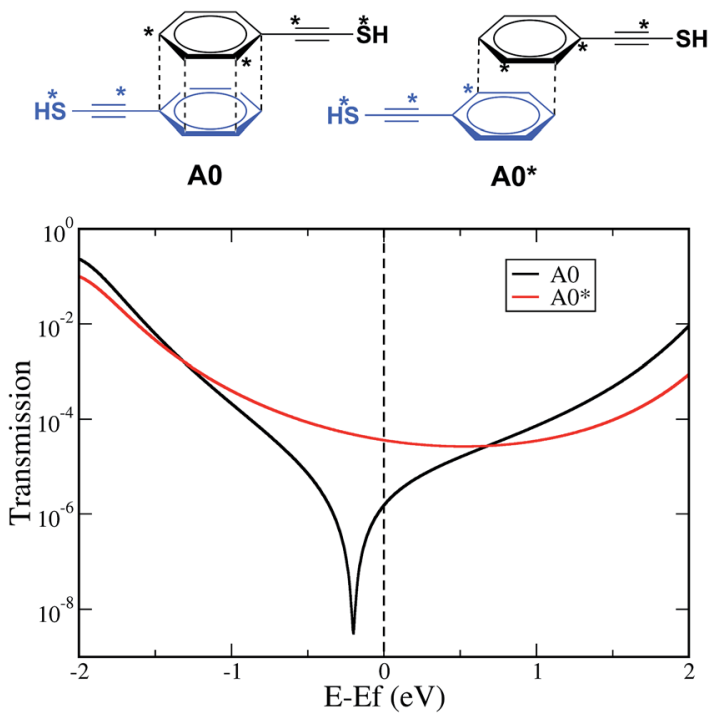

Fig. 2 Top: two selected structures of system A. The molecules are even alternate hydrocarbons and are marked with stars to show when alike (low) or disjoint (high) coupling is expected. Bottom: transmission through the two selected structures of system $A$. 
stars, we say there is alike coupling and destructive interference and when only one is starred we have disjoint coupling and no interference. ${ }^{7}$ Fig. 2 (bottom) shows that the transmission through the fully eclipsed structure has a destructive interference near the Fermi energy, which results in lower transmission, while the dislocated structure has higher transmission near Fermi energy without interference, in agreement with previous results. ${ }^{6}$

In addition, the full range of the transmission, thermopower and power factor are calculated as a function of translation along the long $(x)$ and short $(y)$ axes of the molecule. There is low transmission around $(0.0,0.0)$ in the transmission map, caused by the destructive interference as well as around $(2.0,0.0)$. The high values of thermopower always arise in the vicinity of the destructive interference. So, the whole thermopower map is almost the complementary map of transmission, shown in Fig. 3(b). The sign of thermopower changes with the translation of molecules (positive in red and negative in green). Due to the large

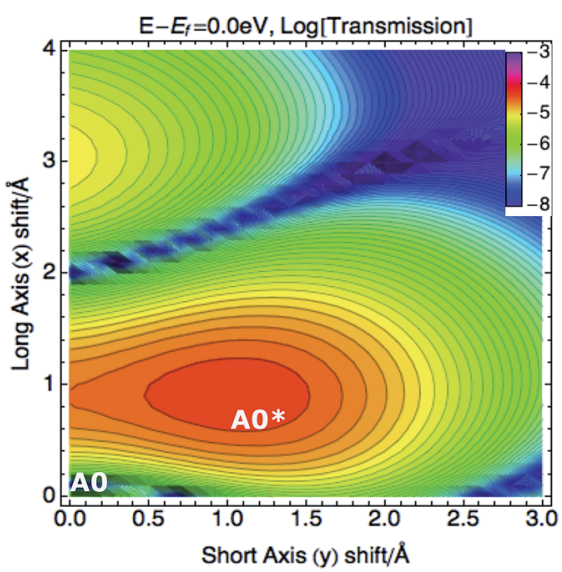

(a)

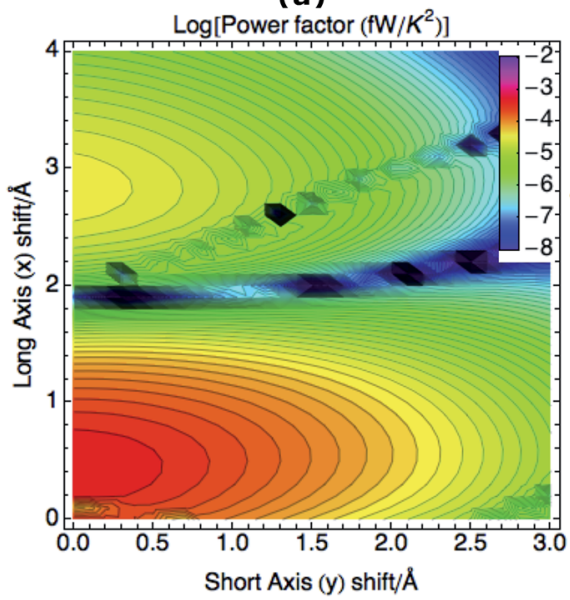

(c)

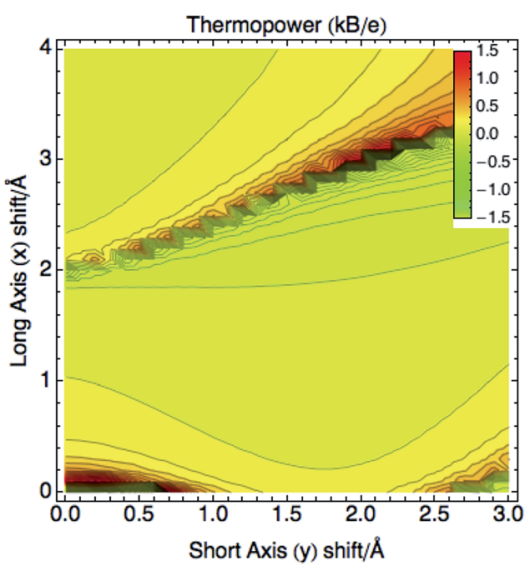

(b)

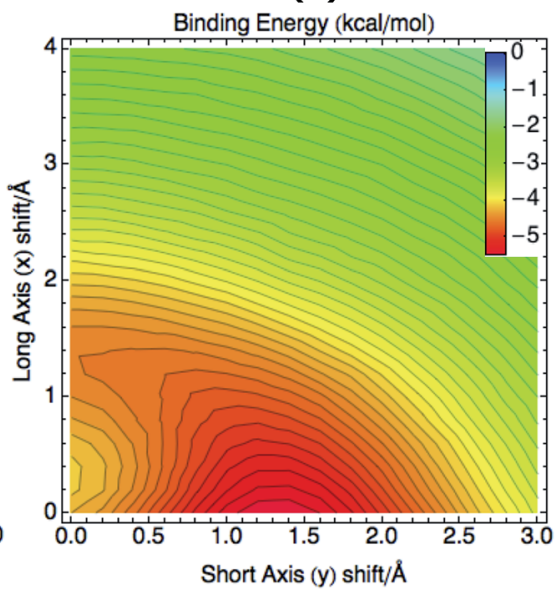

(d)

Fig. 3 Full spatial maps of the transmission (a), thermopower (b), power factor (c), binding energy (d) as a function of translation at the Fermi energy for A. 
thermopower, the areas near $(0.0,0.0)$ which show low transmission, now have a high power factor $S^{2} G$.

The binding energies were calculated as the difference between the total energies of the $\pi$-stacked molecules and the isolated molecules, with van der Waals correction included. As we focus on the $\pi-\pi$ stacking interaction, we only translate molecules along the positive direction of $x$ and $y$-axis, avoiding transport from the triple bond on one molecule into the ring of the other. Consequently, the binding energy map is not the global potential surface. Fig. 3(d) shows that a local minimum is around $(0.0,1.4)$. Although the thermopower $S$ and power factor $S^{2} G$ have relatively high values at the $(0.0,0.0)$ position, the fully eclipsed structure is not the most stable geometry.

\subsection{System B}

In an effort to improve the transmission and thermoelectric properties, the conjugation length of molecules is increased by using anthracene rings instead of benzene rings in the $\pi$-stacked system. There are now three groups of fully eclipsed structures and dislocated structures as the rings are shifted with respect to each other, as shown in Fig. 4 (left). Starring arguments can be used again to predict interference features near the Fermi energy. All three groups that exhibit alike coupling have low transmission from destructive interference near the Fermi energy, while the structures with disjoint coupling have high transmission as shown in Fig. 4 (right). The interference features are effectively "switched on" and "switched off" by translating the relative positions of two molecules. However, the interference positions gradually shift away from the Fermi energy as the system is changed from B0 to B1, B2.

Fig. 5(a) shows that the transmission of system $B$ is generally higher than system A, since there are larger $\pi-\pi$ ring couplings. All the disjoint structures
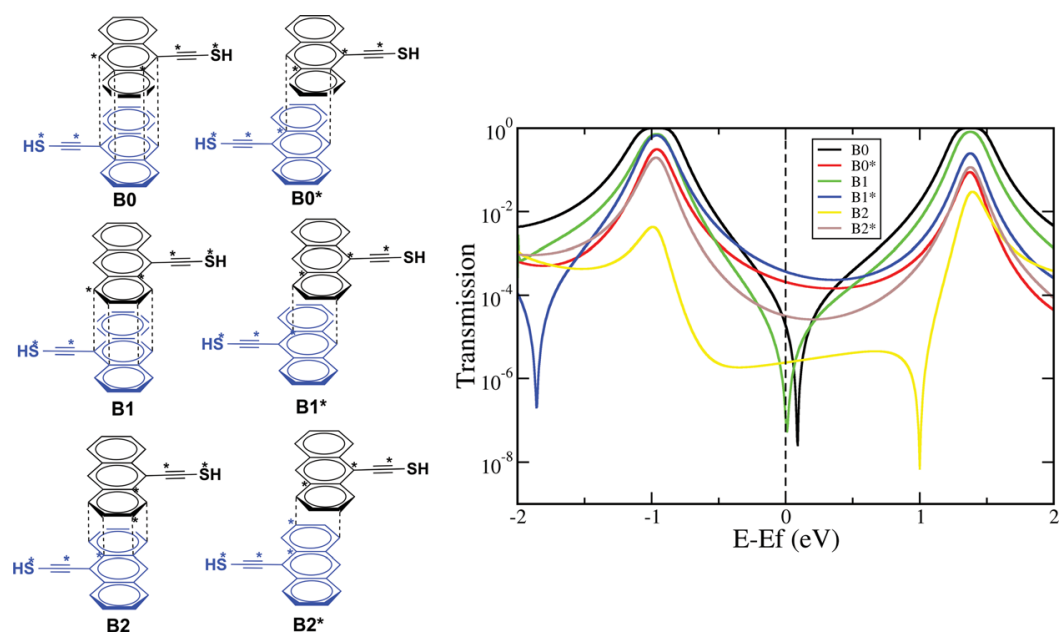

Fig. 4 Left: three groups of selected structures of system B. The molecules are even alternate hydrocarbons and are marked with stars to show when alike (low) or disjoint (high) coupling is expected. Right: transmission through these selected structures of system B. 


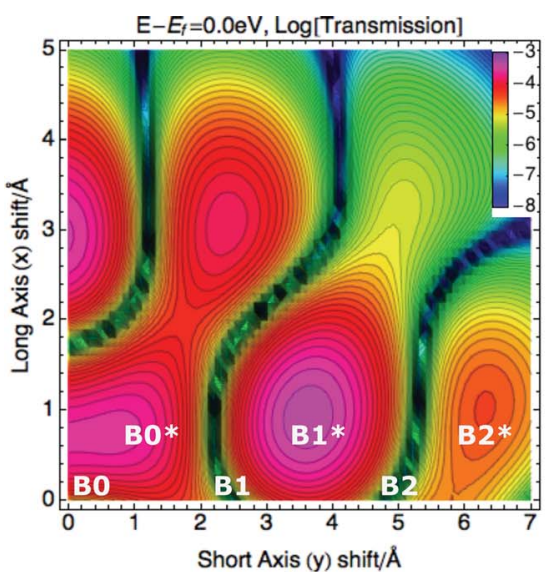

(a)

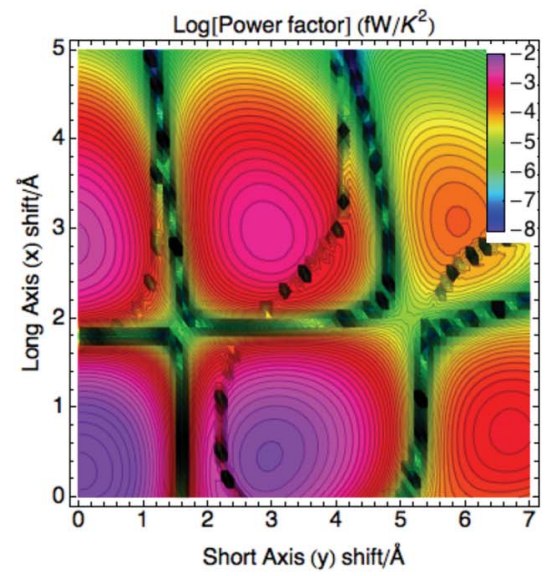

(c)

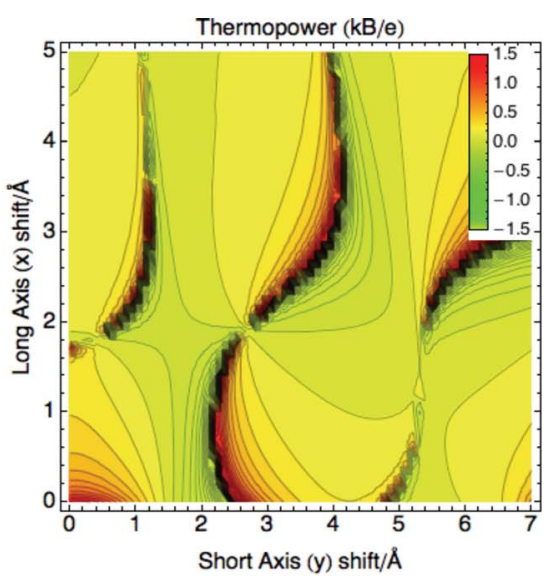

(b)

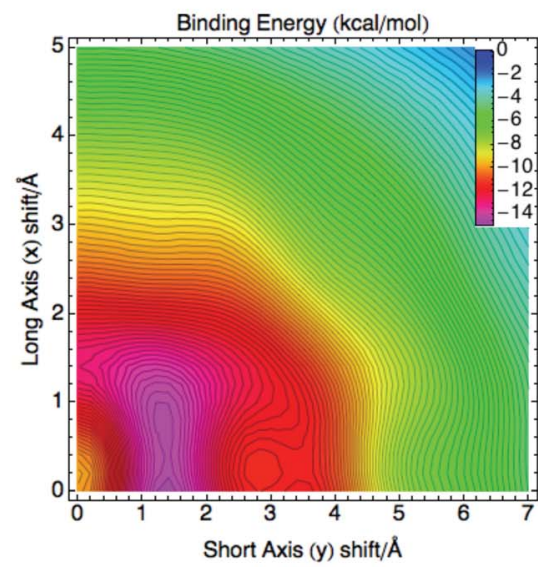

(d)

Fig. 5 Full spatial maps of the transmission (a), thermopower (b), power factor (c), and binding energy (d) as a function of translation at the Fermi energy for system $\mathrm{B}$.

(B0*, B1*, B2*) can be found in the local maxima in the full transmission map, while the "alike" couplings (B0, B1, B2) are located in the low transmission areas. Fig. 5(b) shows more regions with a high thermopower as there are more geometries dominated by destructive interference effects. The sign of the thermopower at these regions switches from positive to negative, as the interference positions shift with the translation. However, the thermopower at the geometries around the fully eclipsed structure of system B all exhibit positive value, which is different from the results of system A. The whole power factor map is dramatically enhanced in the system of anthracene rings, especially at the geometries around the fully eclipsed structure, shown in Fig. 5(c). As the positions of the two molecules are translated along the $y$-direction, the power factor is modulated and periodically presents local maxima. Interestingly, the power factor exhibits very low values along $y=1.6$, which is obviously problematic if we are looking for a large thermoelectric response. The binding energy of system B approaches a local minimum at $(0.0,1.4)$, consistent with what is observed for system A. Again, this 
minimum binding energy position is not the optimal position for the thermopower and power factor. Actually, the minimum energy structure has a very low power factor. In contrast, the fully eclipsed structure of system B has good thermopower value and high power factor and exhibits a good thermoelectric response.

\subsection{System C}

The final question we have in our investigation of the role of conjugation length is: does a mixed combination of an anthracene ring and a benzene ring work better? Fig. 6 (left) shows how the starring assignment works in system C. The transmission results change due to the symmetry breaking. There is destructive interference between the resonances from the highest occupied orbital and lowest unoccupied orbital for the structure $\mathrm{C} 0$, as predicted by the starring rules, but the position is shifted away from the Fermi energy. As the systems are translated along the short axis $(y)$ to $\mathrm{C} 1$, the interference position shifts back to the Fermi energy. Both $\mathrm{C} 0 *$ and $\mathrm{C} 1$ * have high transmission without interference.

Fig. 7(a) shows that the total transmission through system $\mathrm{C}$ is generally weaker than system $\mathrm{B}$. The geometries around $(0.0,0.0)$ exhibit high transmission and a high power factor, but a low thermopower as the interference feature is no longer so close to the Fermi energy. Interestingly, the power factor for system $\mathrm{C}$ also has a very low value along $y=1.6$, which is similar to system $\mathrm{B}$. The local minimum of binding energy for system $\mathrm{C}$ is around $(0.0,0.8)$, shown in Fig. $7(\mathrm{~d})$. However, the geometry of system $\mathrm{C}$ at $(0.0,0.0)$ is more stable than the fully eclipsed structure of system A and exhibits a high transmission and power factor.

\subsection{System D}

Another way to modify the conductance and change the binding energy is to add substituents. Here, we investigate the conductance and thermoelectric response of $\pi$-stacked systems substituted by cyano groups or the combination of a hydroquinone and a quinone. The components of the transmission between pairs
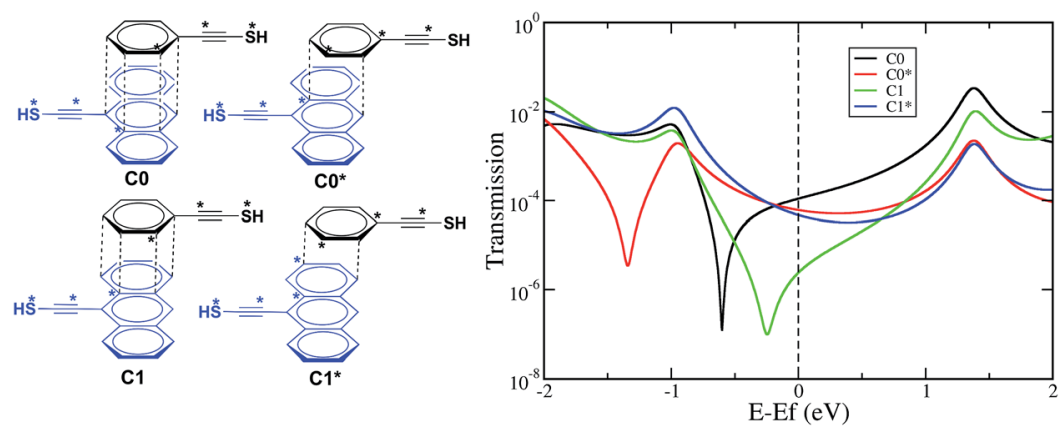

Fig. 6 Left: two groups of selected structures of system C. The molecules are even alternate hydrocarbons and are marked with stars to show when alike (low) or disjoint (high) coupling is expected. Right: transmission through these selected structures of system C. 


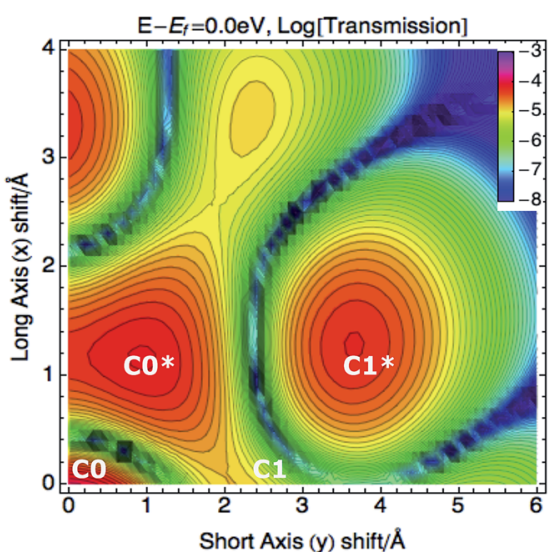

(a)

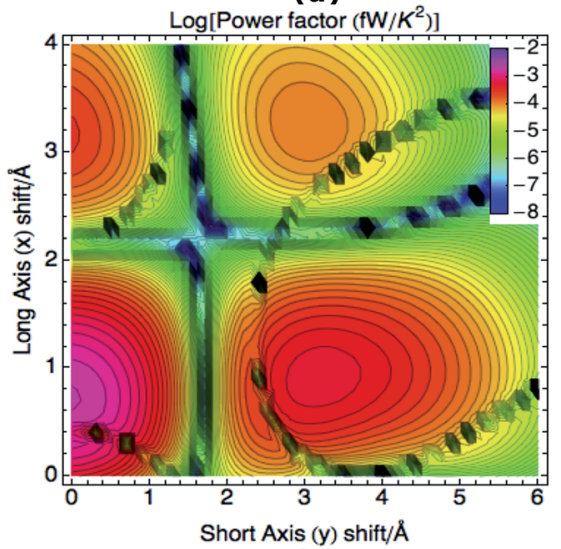

(c)

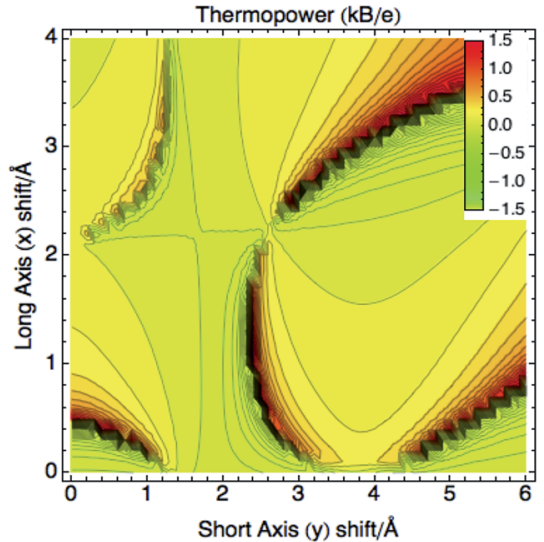

(b)

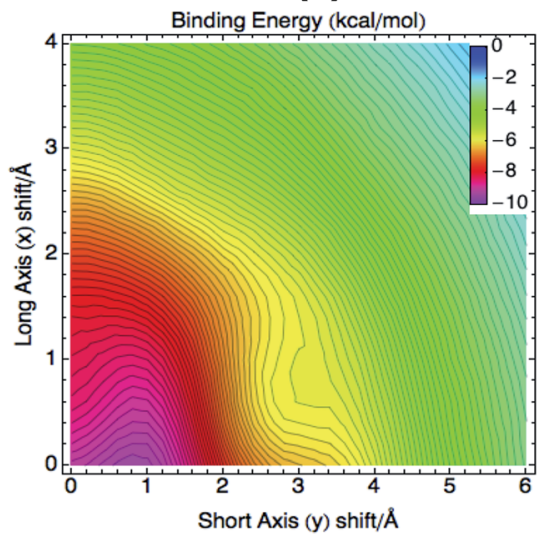

(d)

Fig. 7 Full spatial maps of the transmission (a), thermopower (b), power factor (c), and binding energy (d) as a function of translation at the Fermi energy for system $C$.

of atoms in the molecule, known as the local transmission ${ }^{20}$ is calculated, in order to illustrate the roles of the substituents in the electron transport.

The starring rules cannot be used in the structures with substituents, however, we still consider the two typical geometries in the substituted systems for comparison with system A. Fig. 8 shows that the total transmission of the fully eclipsed structure exhibits destructive interference, similar to the result of system A0. However, at the Fermi energy, the total transmission through system D0 is higher than system A0. When the local transmission at the Fermi energy is calculated, it is found that several extra paths are added by the cyano group, and these contribute to the total transmission. The dislocated structure (D0*), with two sites eclipsed, exhibits high transmission near the Fermi energy and an absence of destructive interference. However, we see less influence of the cyano group, in terms of extra paths in the local transmission through D0* (Fig. 8).

The full spatial map of transmission (Fig. 9) at the Fermi energy shows high values around $(0.0,0.0)$, unlike that seen for system A. There are also more interference areas in the transmission map, compared with system A. This means 


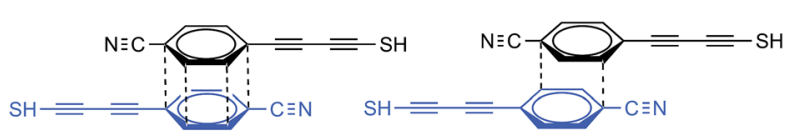

D0

D0*
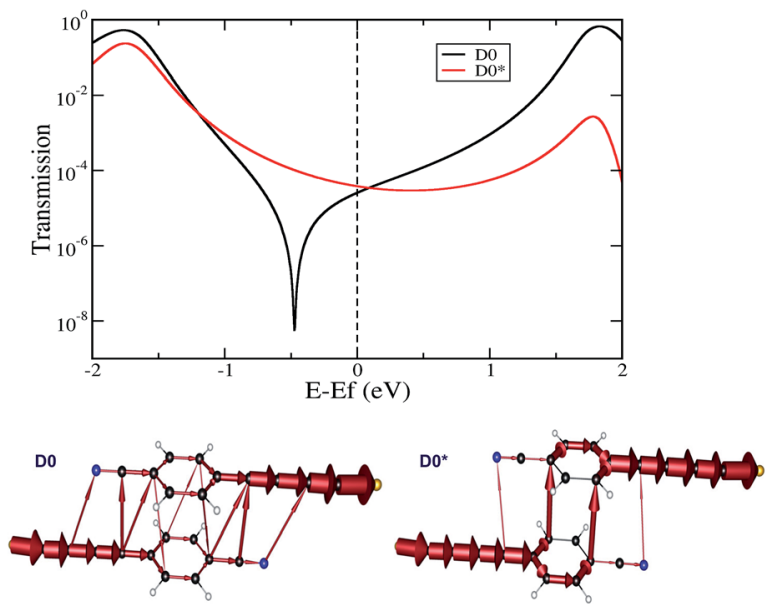

Fig. 8 Top: two selected structures of system D. Middle: total transmission through the two selected structures of system D. Bottom: the local transmission at the Fermi energy through DO and DO*.

that there are also more regions with high thermopower. The most stable position of system $\mathrm{D}$ is located at $(0.0,1.4)$ in the binding energy map, similar to system A. There is, however, an additional local minimum at $(1.6,0.0)$. The power factor is low at both of these two minima areas, but has a high value at the geometries around the fully eclipsed structure, just like system A.

\subsection{System E}

The final system we consider comprises hydroquinone as a donor and quinone as an acceptor. Two selected structures E0 and E0* are shown in Fig. 10 (top), in order to compare with system A. The total transmission of the fully eclipsed structure E0 and the dislocated structure E0* are calculated and shown in Fig. 10 (middle). The transmission exhibits a high value in the structure of E0, and a destructive interference feature is present far from the Fermi energy. E0* shows high transmission without interference features across a wide energy range. The local transmission of system E doesn't indicate any extra paths from hydroquinone and quinone, shown in Fig. 10 (bottom). However, the local transmission of E0 and $\mathrm{E}^{*}$ both show several sites favoured by the overlap of the structure, resulting in their high transmission at the Fermi energy.

There is a region around the structure E0 which exhibits very high transmission in the full spatial map (Fig 11(a)). Fig. 11(b) shows that almost all the geometries exhibit a negative thermopower, except some limited regions with a high positive thermopower. The power factor at the geometries around E0 is significantly enhanced and form local maxima, shown in Fig. 11(c). The binding energies are shown in Fig. 11(d) as a function of $(x, y)$ displacement. Interestingly, 


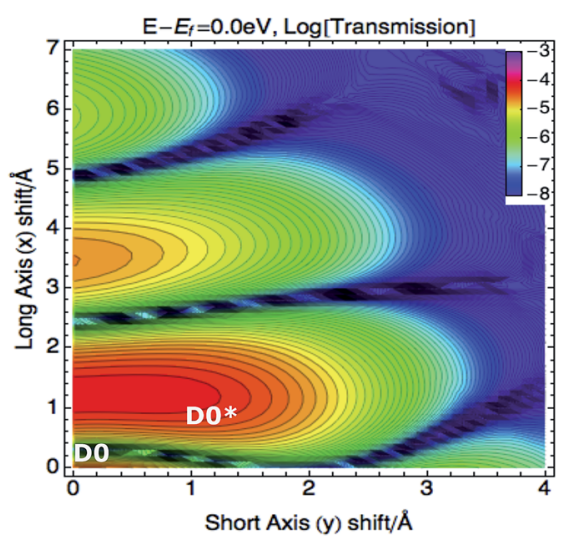

(a)

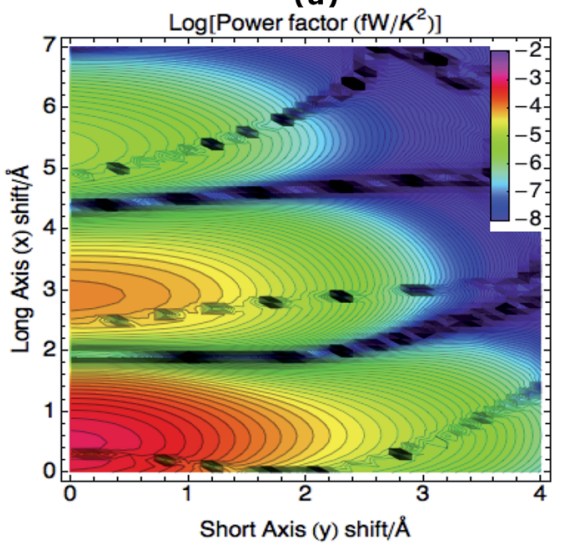

(c)

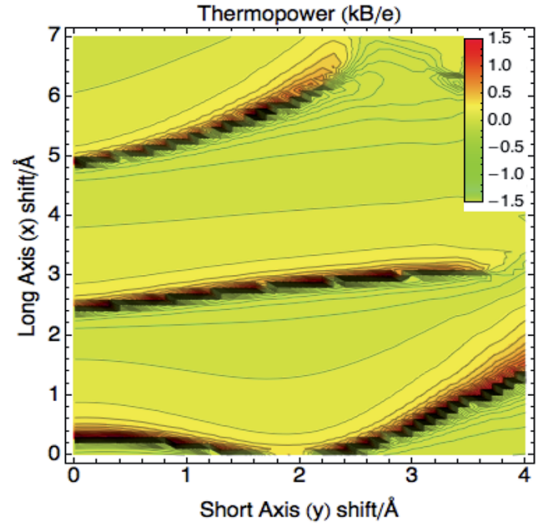

(b)

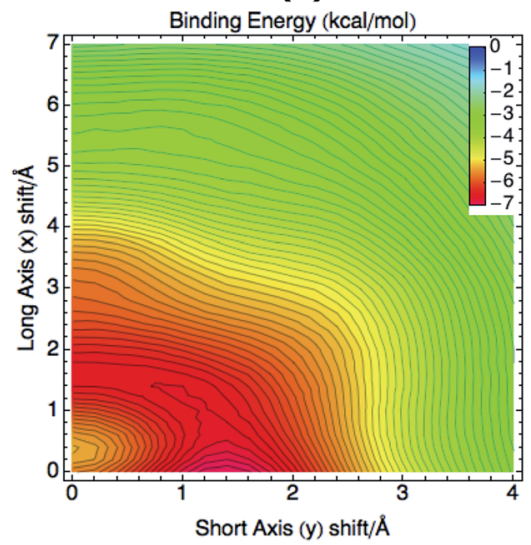

(d)

Fig. 9 Full spatial maps of the transmission (a), thermopower (b), power factor (c), and binding energy (d) as a function of translation at the Fermi energy for system $D$.

the minimum binding energy lies at $(0.0,0.0)$. The fully eclipsed structure of quinhydrone E0 is the most stable structure in all of these geometries, which is different from the former $\pi$-stacked systems. The transmission and power factor of quinhydrone both have high values at $(0.0,0.0)$. The fully eclipsed structure of quinhydrone that has the minimum energy and exhibits a high power factor, making it a possible candidate for a thermoelectric device. While the quinhydrone is a relatively simple donor-acceptor system, it is envisaged that other donor-acceptor pairs could also be explored by this method.

\subsection{Discussion}

While the systems considered here are somewhat artificial, they neatly illustrate a number of points for understanding transport through $\pi$-stacked systems. The conclusions that should be drawn are different for systems where $\pi$-stacked structures form by accident, from those where $\pi$-stacked structures are formed by design.

First we consider the case where aggregation in the junction results in the accidental formation of $\pi$-stacked structures. In a system without substituents, 

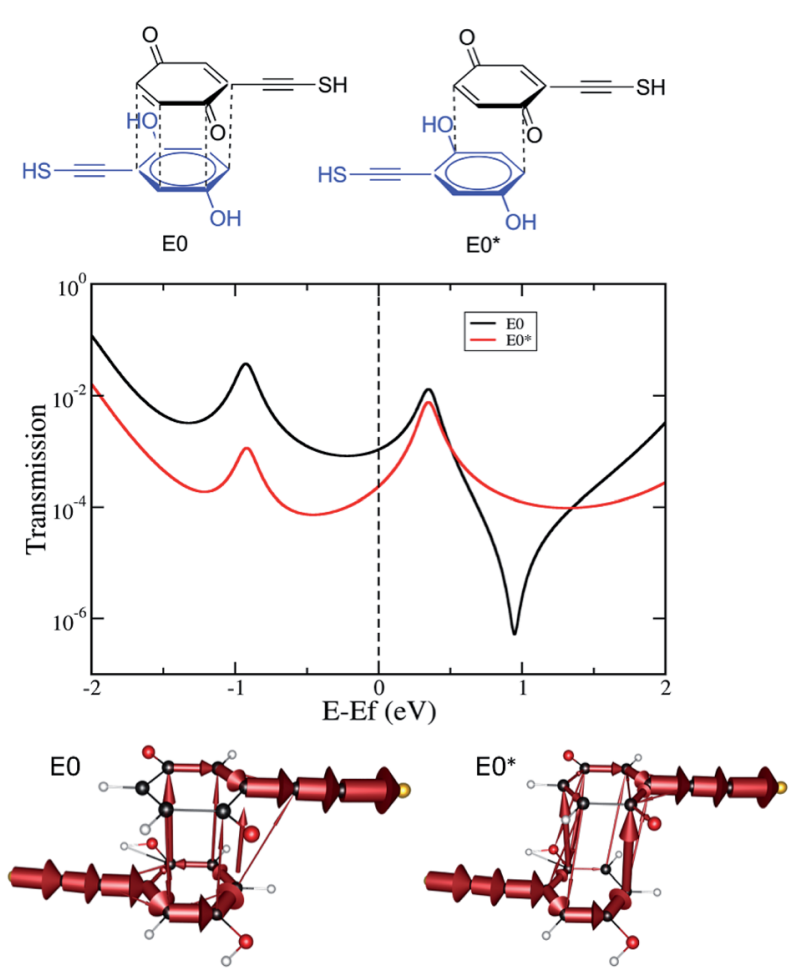

Fig. 10 Top: two selected structures of system E. Middle: total transmission through the two selected structures of system E. Bottom: the local transmission at the Fermi energy through EO and EO*.

the binding energy indicates that the fully eclipsed structures are unlikely to form preferentially but rather some dislocated structure. This means that interference effects are unlikely to dominate and consequently the transport is measurable and the thermopower is not at a maximum. It may be that as these structures are pulled, for example in an STM-break junction experiment, the structure might move closer to or further from an interference feature and thus the transmission decreases or increases and the thermopower increases or decreases.

Substituents can have three effects, they can simply shift the transport resonances (the effect most commonly considered when they are described as electron donating or withdrawing groups), but they can also introduce additional pathways for transport and change the relative stability of different configurations. The case of the system D illustrated how cyano groups can provide an extra pathway and shift interference features. This case is important for considering the transport properties of accidentally formed $\pi$-stacked structures where the molecules involved have two binding groups. In this case, one binding group will be bound to each electrode and the other can be providing additional pathways and shifting interference features.

Using substituents to change the relative stability of different geometries becomes relevant when we seek to design optimal $\pi$-stacked structures. Modifying the system to a quinone and hydroquinone clearly (and somewhat 


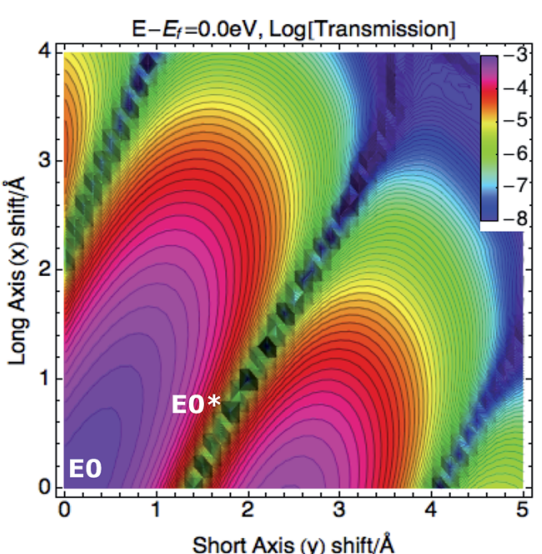

(a)

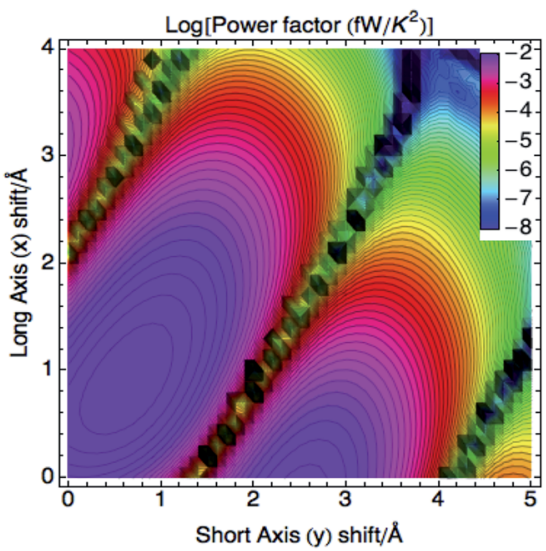

(c)

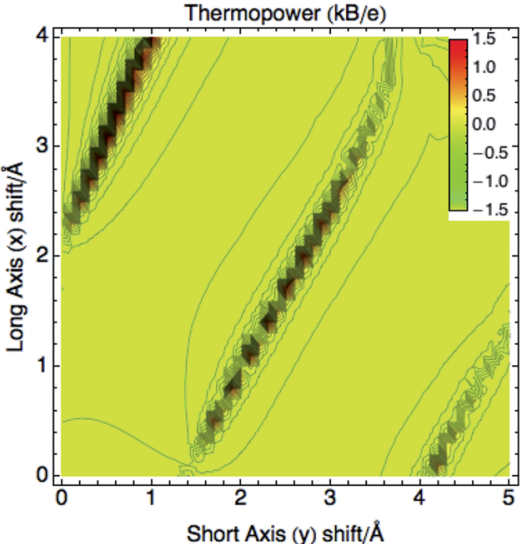

(b)

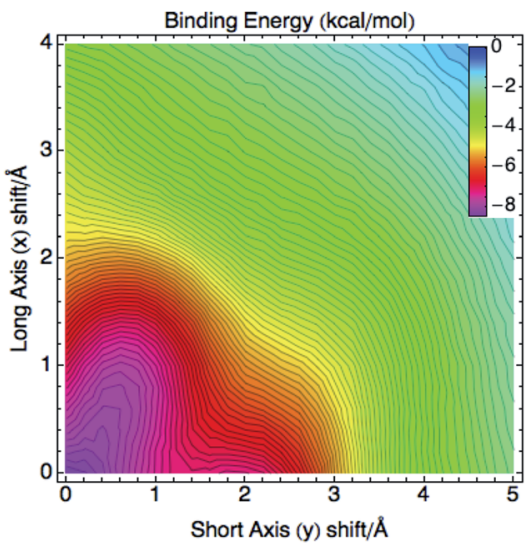

(d)

Fig. 11 Full spatial maps of the transmission (a), thermopower (b), power factor (c), and binding energy (d) as a function of translation at the Fermi energy for system $\mathrm{E}$.

obviously) results in the fully eclipsed structure being favoured as two hydrogen bonds can form and we then can achieve the result that the most stable structure also maximises the thermoelectric response. The fully eclipsed structures could also be formed by binding the two sides together in a cyclophane; however, in this case the potential advantage of a non-bonded system for reducing heat transport is lost. While it was not explored in this paper, it has been shown that substitution can shift the minima across a considerable region of the map in conjugated systems ${ }^{21}$ suggesting that we really can pick out regions of interest and design molecules to form these structures.

\section{Conclusions}

We found that the transmission and power factor $\left(S^{2} G\right)$ are both enhanced by increasing the conjugation length or adding substituent groups. In particular, the $\pi$-stacked system of two anthracene rings exhibits a good thermopower value and high power factor, predicting that an increased conjugation length can 
dramatically enhance the thermoelectric response. The local transmission of system D shows that several extra paths are added by the cyano group, which increases the total transmission at the Fermi energy. Substituents can also change the structures that will be formed in junctions, with the local minimum binding energy positions of system A and system B both lying at $(0.0,1.4)$, while the most stable structure of quinhydrone is at $(0.0,0.0)$. The fully eclipsed structure of quinhydrone is the most stable system that could exhibit high transmission and power factor in these five $\pi$-stacked systems.

\section{Acknowledgements}

We would like to thank Mikkel Strange, Jacob Lykkebo and Tim Hansen for helpful discussions. This work was supported through funding from the European Research Council under the European Union's Seventh Framework Program (FP7/2007- 2013)/ERC Grant Agreement no. 258806 and The Danish Council for Independent Research Natural Sciences.

\section{References}

1 B. Giese, Annu. Rev. Biochem., 2002, 71, 51-70.

2 M. D. Watson, F. Jckel, N. Severin, J. P. Rabe and K. Mllen, J. Am. Chem. Soc., 2004, 126, 1402-1407.

3 S. Wu, M. T. Gonzalez, R. Huber, S. Grunder, M. Mayor, C. Schonenberger and M. Calame, Nat. Nanotechnol., 2008, 3, 569-574.

4 S. Martin, I. Grace, M. R. Bryce, C. Wang, R. Jitchati, A. S. Batsanov, S. J. Higgins, C. J. Lambert and R. J. Nichols, J. Am. Chem. Soc., 2010, 132, 9157-9164.

5 S. T. Schneebeli, M. Kamenetska, Z. Cheng, R. Skouta, R. A. Friesner, L. Venkataraman and R. Breslow, J. Am. Chem. Soc., 2011, 133, 2136-2139.

6 G. C. Solomon, C. Herrmann, J. Vura-Weis, M. R. Wasielewski and M. A. Ratner, J. Am. Chem. Soc., 2010, 132, 7887-7889.

7 G. C. Solomon, J. Vura-Weis, C. Herrmann, M. R. Wasielewski and M. A. Ratner, J. Phys. Chem. B, 2010, 114, 14735-14744.

8 J. P. Bergfield and C. A. Stafford, Nano Lett., 2009, 9, 3072-3076.

9 P. Reddy, S.-Y. Jang, R. A. Segalman and A. Majumdar, Science, 2007, 315, 15681571.

10 K. Baheti, J. A. Malen, P. Doak, P. Reddy, S.-Y. Jang, T. D. Tilley, A. Majumdar and R. A. Segalman, Nano Lett., 2008, 8, 715-719.

11 J. A. Malen, P. Doak, K. Baheti, T. D. Tilley, R. A. Segalman and A. Majumdar, Nano Lett., 2009, 9, 1164-1169.

12 S. K. Yee, J. A. Malen, A. Majumdar and R. A. Segalman, Nano Lett., 2011, 11, 4089-4094.

13 A. Tan, J. Balachandran, S. Sadat, V. Gavini, B. D. Dunietz, S.-Y. Jang and P. Reddy, J. Am. Chem. Soc., 2011, 133, 8838-8841.

14 S. Datta, Quantum Transport: Atom to Transistor, Cambridge University Press, New York, 1st edn, 2005.

15 J. Enkovaara, C. Rostgaard, J. J. Mortensen, J. Chen, M. Duak, L. Ferrighi, J. Gavnholt, C. Glinsvad, V. Haikola, H. A. Hansen, H. H. Kristoffersen, M. Kuisma, A. H. Larsen, L. Lehtovaara, M. Ljungberg, O. Lopez-Acevedo, 
P. G. Moses, J. Ojanen, T. Olsen, V. Petzold, N. A. Romero, J. Stausholm-Mller, M. Strange, G. A. Tritsaris, M. Vanin, M. Walter, B. Hammer, H. Hkkinen, G. K. H. Madsen, R. M. Nieminen, J. K. Nrskov, M. Puska, T. T. Rantala, J. Schitz, K. S. Thygesen and K. W. Jacobsen, J. Phys.: Condens. Matter, 2010, 22, 253202.

16 A. Tkatchenko and M. Scheffler, Phys. Rev. Lett., 2009, 102, 073005.

17 T. Frauenheim, G. Seifert, M. Elstner, T. Niehaus, C. Khler, M. Amkreutz, M. Sternberg, Z. Hajnal, A. D. Carlo and S. Suhai, J. Phys.: Condens. Matter, 2002, 14, 3015.

18 A. Pecchia and A. D. Carlo, Rep. Prog. Phys., 2004, 67, 1497.

19 L. Salem, The Molecular Orbital Theory of Conjugated Systems, ed. W. A. Benjamin, New York, 1st edn, 1966.

20 G. C. Solomon, C. Herrmann, H. Thorsten, V. Mujica and M. A. Ratner, Nat. Chem., 2010, 2, 223-228.

21 J. Vura-Weis, M. A. Ratner and M. R. Wasielewski, J. Am. Chem. Soc., 2010, 132, 1738-1739. 